\title{
Dynamics of the conservative and dissipative spin-orbit problem
}

\author{
Alessandra Celletti \\ Dipartimento di Matematica, Università di Roma Tor Vergata, Via della Ricerca \\ Scientifica 1, I-00133 Roma (Italy), celletti@mat.uniroma2.it \\ Claude Froeschlé \\ Observatoire de Nice, B.P.229,06304 Nice Cedex4 (France), claude@obs-nice.fr \\ Elena Lega \\ Observatoire de Nice, B.P.229,06304 Nice Cedex 4 (France), claude@obs-nice.fr
}

Keywords: Spin-orbit coupling, resonances, dissipative forces.

\begin{abstract}
We investigate the dynamics of the spin-orbit coupling under different settings. First we consider the conservative problem, and then we add a dissipative torque as provided by MacDonald's or Darwin's models. By means of frequency analysis and of the computation of the maximum Lyapunov indicator we explore the different dynamical behaviors associated to the main resonances. In particular we focus on the 1:1 and 3:2 resonances in which the Moon and Mercury are actually trapped.
\end{abstract}




\section{Introduction}

One of the most fascinating puzzles of Celestial Mechanics concerns the different behavior of satellites and planets under a spin-orbit interaction. More precisely, consider the coupling between the revolution of a satellite around a primary and its rotation about an internal spin-axis. As is well known, most of the evolved bodies of the Solar System (including the Moon) are trapped in a 1:1 resonance, i.e. they always point the same hemisphere to the host primary. A unique exception is provided by Mercury, which is currently captured in a 3:2 resonance (i.e., after 2 revolutions around the Sun, Mercury completes 3 rotations). The different occurrence of the 1:1 and 3:2 resonances is subject to the variations of some key parameters which enter the model, like the orbital eccentricity, the oblateness parameter and the dissipation factor (compare with [1], [2], [4], [6] and [10], [15], [16] for a discussion of capture into resonance).

In the present work we consider a simple spin-orbit model by assuming that the satellite moves on a Keplerian orbit and that it rotates around a principal axis with zero obliquity (see, e.g., [1] for further details). Under these assumptions, the equation of motion is given by a second-order time-dependent differential equation in the angle describing the relative orientation of the shorter principal axis with respect to a preassigned direction. However such conservative model is not always adequate to explain the dynamics of the spin-orbit coupling, since the tidal torque induced by the internal non-rigidity of the satellite might affect the dynamics. To this end, we consider two different forms of tidal dissipation as provided by the classical MacDonald's and Darwin's torques (see, e.g., [7], $[8]$ ). MacDonald dissipation ([13]) is characterized by a phase lag depending linearly on the angular velocity, while Darwin's torque ([5]) uses a Fourier development of the tidal potential, assigning to each component a constant amplitude. 
The conservative and dissipative models are investigated through frequency analysis ([11], [12]) and by means of the computation of the largest Lyapunov indicator (see, e.g., [18]; see also [3] for a discussion of similar problems in the framework of the dissipative standard map). Notice that we are not interested in the precise value of the maximum Lyapunov exponent, but only in its value at a given time (maximum Lyapunov indicator). The above techniques confirm that the chance of a given resonance is strongly affected by the eccentricity and by the dissipation factor. In particular, the synchronous commensurability is most likely for small eccentricities, while the role of the 3:2 resonance is more important for eccentricities comparable to that of Mercury. As far as the dissipation is concerned, we remark that the capture into a resonant regime increases as the dissipation gets stronger, suggesting that in the early stages of the Solar System the dissipative contribution might have played a key role in the selection of the resonances.

\section{The conservative spin-orbit model}

Let $S$ be a triaxial satellite orbiting around a central body, say $P$, and rotating about an internal spin-axis. Let $T_{\text {rev }}$ and $T_{\text {rot }}$ be the periods of revolution and rotation of the satellite.

Definition. Let $p$ and $q$ be positive integers. A spin-orbit resonance of order $p: q$ occurs whenever

$$
\frac{T_{r e v}}{T_{r o t}}=\frac{p}{q}
$$

Our framework is provided by a spin-orbit model obtained by assuming the following hypotheses (see [1]): 
i) the satellite moves on a Keplerian orbit around the primary (i.e., there are no secular perturbations on the elliptic elements);

ii) the spin-axis coincides with the smallest physical axis (i.e., it coincides with the largest principal direction);

iii) the spin-axis is perpendicular to the orbit plane (i.e., the obliquity angle is zero);

$i v$ ) any dissipative force (including the tidal torque due to the internal non-rigidity of the satellite) as well as other gravitational perturbations are neglected.

The equation of motion describing the spin-orbit model under the assumptions $i)-i v$ ) can be expressed in terms of the following quantities: with reference to the Keplerian orbit of the satellite, let $a, r, f$ be, respectively, the semimajor axis, the orbital radius and the true anomaly. Assuming that the satellite has an ellipsoidal shape, let $A<B<C$ be the principal moments of inertia and let $x$ be the angle between the longest axis of the ellipsoid and the pericentre line. Then, the equation of motion is given by (see [1], $[7])$

$$
\ddot{x}+\frac{3}{2} \frac{B-A}{C}\left(\frac{a}{r}\right)^{3} \sin (2 x-2 f)=0 .
$$

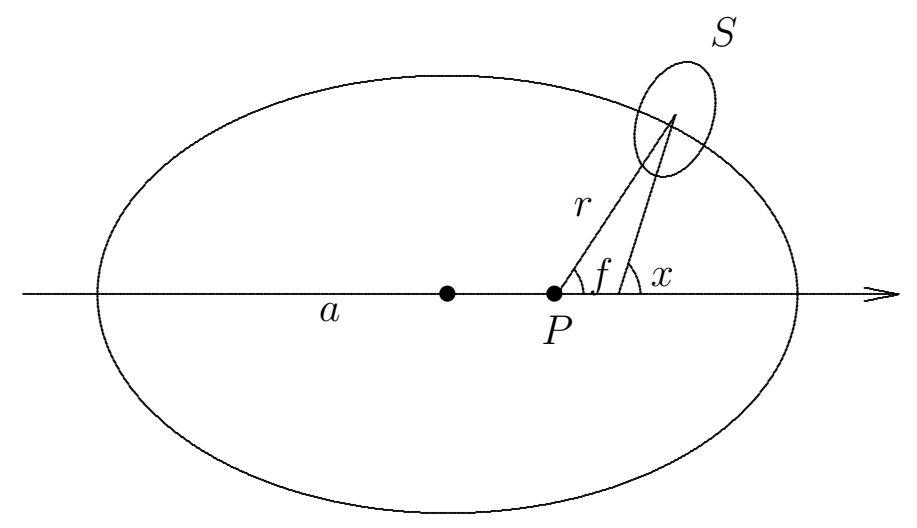

\section{Remarks.}


a) The instantaneous orbital radius $r$ and the true anomaly $f$ are Keplerian functions of the time. The differential equation (1) depends periodically on the time, through the Keplerian elements $r$ and $f$ with period $2 \pi$. Therefore a periodic orbit associated to (1) must have a period multiple of $2 \pi$. More precisely, a spin-orbit resonance of order $\frac{p}{q}$ corresponds to a periodic orbit $x=x(t)$ such that

$$
x(t+2 \pi q)=x(t)+2 \pi p
$$

implying that after $q$ revolutions around the primary, the satellite makes $p$ rotations about the spin-axis. For a 1:1 or synchronous spin-orbit resonance, the angle $x$ is always oriented according to the direction of the instantaneous orbital radius, so that the periods of rotation and of revolution are equal; as a consequence, the satellite always points the same face to the host body.

b) The parameter $\varepsilon \equiv \frac{3}{2} \frac{B-A}{C}$ represents the equatorial oblateness of the satellite; its value is zero whenever $A=B$, namely when the satellite is symmetric on the equator. In this case the system is trivially integrable. The true values of the parameter $\varepsilon$ for the Moon and Mercury are, respectively, $\varepsilon=3.45 \cdot 10^{-4}$ and $\varepsilon=1.5 \cdot 10^{-4}$.

c) The orbital radius and the true anomaly depend on the eccentricity $e$ and, in particular, they reduce to $r=a=$ const and $f=n t+$ const ( $n$ being the mean motion) whenever $e=0$. Therefore, in case of circular orbits (1) reduces to the integrable equation $\ddot{x}+\varepsilon \sin (2 x-2 n t+2$ const $)=0$.

d) The orbital radius and the true anomaly are related to the eccentric anomaly $u$ by the well-known relations (see, e.g., [14])

$$
r=a(1-e \cos u)
$$




$$
f=2 \arctan \left(\sqrt{\frac{1+e}{1-e}} \tan \frac{u}{2}\right)
$$

whereas $u$ is related to the mean anomaly $\ell$ through Kepler's equation $\ell=u-e \sin u$.

\section{MacDonald's and Darwin's dissipative models}

Different forms of the dissipation might be adopted to express a tidal torque acting on a satellite under a spin-orbit coupling. Here we focus on two dissipative forces widely known in the literature under the names of MacDonald's and Darwin's torques (see [7], [8], [14]). The first one ([13]) assumes a phase lag which depends linearly on the angular

velocity, while the latter ([5]) Fourier decomposes the tidal potential, assigning to each component a constant amplitude. More precisely, following [4] we write the equation of motion under a MacDonald torque as

$$
\ddot{x}+\frac{3}{2} \frac{B-A}{C}\left(\frac{a}{r}\right)^{3} \sin (2 x-2 f)=-K[\Omega(e) \dot{x}-N(e)],
$$

where

$$
\begin{aligned}
& \Omega(e) \equiv \frac{1}{\left(1-e^{2}\right)^{9 / 2}}\left(1+3 e^{2}+\frac{3}{8} e^{4}\right) \\
& N(e) \equiv \frac{1}{\left(1-e^{2}\right)^{6}}\left(1+\frac{15}{2} e^{2}+\frac{45}{8} e^{4}+\frac{5}{16} e^{6}\right),
\end{aligned}
$$

while $K$ is the dissipative constant depending on the physical and orbital characteristics of the satellite. In particular, one has:

$$
K \equiv 3 n \frac{k_{2}}{\xi Q}\left(\frac{R}{a}\right)^{3} \frac{m_{0}}{m},
$$

where $k_{2}$ is the Love's number, $Q$ is the quality factor, $m$ is the mass of the satellite, $m_{0}$ is the mass of the central body, $R$ is the radius of the satellite, $\xi$ is a structure constant 
Table 1

Some physical and orbital constants for the Moon and Mercury

\begin{tabular}{|c|c|c|}
\hline & Moon & Mercury \\
\hline$k_{2}$ & 0.02 & 0.4 \\
\hline$Q$ & 150 & 50 \\
\hline$m$ & $7.35 \cdot 10^{22} \mathrm{~kg}$ & $3.302 \cdot 10^{23} \mathrm{~kg}$ \\
\hline$m_{0}$ & $5.972 \cdot 10^{24} \mathrm{~kg}$ & $1.99 \cdot 10^{30} \mathrm{~kg}$ \\
\hline$R$ & $1737.5 \mathrm{~km}$ & $2440 \mathrm{~km}$ \\
\hline$\xi$ & 0.392 & 0.333 \\
\hline$a$ & $3.844 \cdot 10^{5} \mathrm{~km}$ & $5.79093 \cdot 10^{7} \mathrm{~km}$ \\
\hline$e$ & 0.0554 & 0.2056 \\
\hline$n$ & $84.002 y r^{-1}$ & $26.0879 y r^{-1}$ \\
\hline
\end{tabular}

such that $C=\xi m R^{2}$. For the Moon and Mercury the numerical values of such quantities are reported in Table 1 . With these values it is $K_{\text {Moon }}=6.43162 \cdot 10^{-7} y r^{-1}$ for the Moon and $K_{\text {Merc }}=8.4687 \cdot 10^{-7} y^{-1}$ for Mercury.

Concerning Darwin's torque, we assume the following expression of the equation of motion $([8],[17])$ :

$$
\ddot{x}+\frac{3}{2} \frac{B-A}{C}\left(\frac{a}{r}\right)^{3} \sin (2 x-2 f)=
$$




$$
\begin{aligned}
& -K\left[W_{-2}(e)^{2} \operatorname{sgn}(x+1)+W_{-1}(e)^{2} \operatorname{sgn}\left(x+\frac{1}{2}\right)+W_{1}(e)^{2} \operatorname{sgn}\left(x-\frac{1}{2}\right)+W_{2}(e)^{2} \operatorname{sgn}(x-1)\right. \\
& \left.+W_{3}(e)^{2} \operatorname{sgn}\left(x-\frac{3}{2}\right)+W_{4}(e)^{2} \operatorname{sgn}(x-2)+W_{5}(e)^{2} \operatorname{sgn}\left(x-\frac{5}{2}\right)+W_{6}(e)^{2} \operatorname{sgn}(x-3)\right],(3)
\end{aligned}
$$

where the coefficients $W_{k}(e)$ depend on the eccentricity through the following expressions:

$$
\begin{aligned}
W_{-2}(e) & =\frac{e^{4}}{24} & W_{-1}(e) & =\frac{e^{3}}{48} \\
W_{1}(e) & =-\frac{e}{2}+\frac{e^{3}}{16} & W_{2}(e) & =1-\frac{5 e^{2}}{2}+\frac{13 e^{4}}{16} \\
W_{3}(e) & =\frac{7 e}{2}-\frac{123 e^{3}}{16} & W_{4}(e) & =\frac{17 e^{2}}{2}-\frac{115 e^{4}}{6} \\
W_{5}(e) & =\frac{845 e^{3}}{48} & W_{6}(e) & =\frac{533 e^{4}}{16} .
\end{aligned}
$$

\section{Exploration of the dynamics}

\subsection{Frequency analysis}

A widespread technique to study the behavior of conservative systems is the socalled frequency map analysis, based on the investigation of the frequency as a function of the perturbing parameter (see [11], [12]). When dealing with a dissipative system it is essential to perform some preliminary iterations in order to let the system evolve toward an attractor; after this transient time (typically we perform 5000 iterations with step-size $2 \pi / 50$ ), we implement frequency analysis in the style of [11] to compute the main frequencies as a function of the perturbing parameter. Euler method, modified in order to be area-preserving, has been used (see Appendix A for a short discussion of the implementation of different integration algorithms). We remark that the transient time can become very large in the weakly dissipative regime (i.e. when the dissipative 
parameter $K$ is close to zero), since the evolution toward the attractor may be very slow.

Let $y \equiv \dot{x}$; with reference to equations (1), (2) or (3), we consider a sample of 3 initial conditions in the $y$-variable, while we always set $x=0: y=0.6$, which corresponds to an initial datum close to the golden sectio $\frac{\sqrt{5}-1}{2} \simeq 0.618, y=1$ associated to the 1:1 resonance, $y=1.5$ for the $3: 2$ resonance (notice that a $p / q$ spin-orbit resonance occurs whenever $y / n=p / q$, where $n$ is the mean motion which has been set to one in our equations). We studied either the conservative and dissipative cases with different values of the dissipation parameter, say from the physical values $K_{M o o n}, K_{M e r c}$ up to $10^{-4}$; notice that it is physically meaningless to consider higher values, since they would exceed the conservative parameter which is about $10^{-4}$ in both cases.

The results are presented through graphs showing the behavior of the frequency $\omega$ versus the perturbing parameter $\varepsilon$ for different values of the dissipation. We remark that our finite time integrations allow to detect the presence of a resonant behavior on that given time interval up to some resonant threshold order, say $K$ (compare with [9]). When a continuum of tori is displayed, it means that the resonances within the given domain have order larger than $K$. Similarly a whole resonant region indicates a regular librational zone around the resonance; as in the previous case, secondary resonances are not detected above a given order. The same comment shall apply for the implementation of the Lyapunov charts provided in the next section.

We now start to deal with the Moon's sample in the conservative setting: for $y=1$ and $x=0$ the motion takes always place close to the $1: 1$ resonance apart for a small interval in the parameter $\varepsilon$ included in $[0.12,0.14]$ (see Figure 1a) where chaotic motion is observed (in agreement with Figure 4 top left). The situation is very similar when 
adding the dissipation, even for the dissipative value $K=10^{-4}$. A different behavior is found for $y=1.5$, since the motion is trapped in a $3: 2$ resonance up to $\varepsilon=0.094$, while after such threshold one finds chaotic motions (see Figure 1b). We report that for the initial condition $y=0.6$, one finds quasi-periodic attractors up to about $\varepsilon=0.065$.

Let us now investigate the case of Mercury starting again with the conservative setting. For $y=1$ the motion is trapped in a 1:1 resonance up to $\varepsilon=0.067$ and for $\varepsilon$ in the interval 0.078-0.114 (see Figure 1c). For $y=1.5$, Mercury is trapped in a 3:2 resonance up to $\varepsilon=0.097$ (see Figure 1d and compare with Figure 4, top right). This situation remains quite similar also in the dissipative case up to $K=10^{-5}$; a different behavior is observed for higher dissipations, say $K=10^{-4}$, since one finds a trapping into a 3:2 resonance only for $\varepsilon$ belonging to the interval 0.04-0.08. Finally, for $y=0.6$ we report that quasi-periodic attractors appear until $\varepsilon=0.054$ in the conservative case as well as in the dissipative case up to $K=10^{-6}$; for $K=10^{-5}$ one finds an invariant curve attractor for a value of $\varepsilon$ slightly smaller, say $\varepsilon=0.053$, while for $K=10^{-4}$ the invariant curve attractor is found up to $\varepsilon=0.052$.

From the analysis of Figure 1 we conclude that the 1:1 resonance appears to be quite robust with respect to the oblateness and to the dissipation for a Moon's like satellite, while its occurrence is comparable to that of the 3:2 resonance as far as Mercury's like satellites are considered.

\subsection{Maximum Lyapunov indicator}

A standard tool to evaluate the chaotic dynamics is the maximum Lyapunov indicator (see, e.g., [18] for a discussion and practical implementations), which provides information 
of the divergence of nearby trajectories. In this section we present some grey scale charts which show in a convenient way the results for the maximum Lyapunov indicator; more precisely, periodic orbits are denoted with black, invariant tori are grey, while chaotic and strange attractors are white. The overall integration time is always set to 1000 iterations with step-size $2 \pi / 50$; moreover, in the dissipative cases we performed 5000 preliminary iterations to get closer to the attractor. Like for the frequency analysis method we complain that the transient time should be much larger in a weakly dissipative regime. However, it is important to stress that we do not aim to forecast the motion over long time scales, but rather to compare the dynamics as the eccentricity is varied.

Figure 2 shows the maximum Lyapunov indicator in the plane $y-x$ for the Moon $(e=$ $0.0549)$ and for Mercury $(e=0.2056)$, setting $\varepsilon=0.1$ and taking $K=0$ (conservative regime) or $K=10^{-4}$. This figure is obtained starting with some initial conditions $\left(x_{0}, y_{0}\right)$ and computing the corresponding Lyapunov exponent, whose value is represented within a grey scale. We remark that in the case of the Moon, the 1:1 resonance is quite big in comparison to the 3:2 and 2:1 resonances; as the dissipative parameter grows the orbits inside the librational islands spiral toward the central resonance and new periodic orbits appear, replacing invariant curves and filling also the chaotic region. Concerning Mercury, it is evident that the situation is quite different, since the size of the $1: 1$ resonance is comparable to that of the 3:2 resonance (see Figure 2, bottom).

It is interesting to compare the Lyapunov indicator maps of Figure 2 (top left and top right) with the computation of the corresponding Poincaré maps at times multiples of $2 \pi$ as in Figure 3 . We considered the conservative case and 200 initial conditions of the $y$-variable, equally spaced between 0.5 and 2.5 (we always set $x=\pi$ ). We fixed $\varepsilon=0.1$ and we selected a step-size equal to $10^{-2}$. With respect to the Lyapunov charts, the Poincaré maps allow to detail with greater precision the structure of the primary and 
secondary resonances, as well as the regions filled by invariant tori.

The effect of an increasing eccentricity is shown in Figure 4, which refers to the conservative case with $e=0.3$ and $e=0.4$. One immediately remarks that the size of the main resonances decreases as the eccentricity increases; indeed, the 1:1, 3:2 and 2:1 resonances are extremely small at $e=0.4$. A similar behavior is observed for non-zero values of the dissipative parameter.

Figure 5 shows the maximum Lyapunov indicator in the $y-\varepsilon$ plane for a fixed $x=0$ and for different values of the dissipative parameter. Both Moon and Mercury eccentricities have been considered.

In agreement with the results obtained so far, we remark that in the case of the Moon the amplitude of the synchronous librational island for a given $\varepsilon$ is definitely bigger than those of the 3:2 and 2:1 resonances; on the contrary, such amplitudes are of the same order of magnitude as far as Mercury's eccentricity is concerned. Notice that white regions correspond to the chaotic separatrix or to strange attractors. The dissipative case with $K=10^{-4}$ has been also considered (Figure 5 , bottom) and shows that most of the dynamics takes place on periodic orbits. It is remarkable that Darwin's form for the dissipation provides results definitely similar to those obtained with the MacDonald friction (compare with Figure 6 and Figure 5, bottom).

\subsection{Occurrences of resonances}

The number of occurrences of $p: q$ resonances is strongly related to the main parameters which mostly influence the dynamics, i.e. the eccentricity and the dissipative parameter. Let us fix the perturbing parameter to $\varepsilon=0.1$; Figure 7 shows the number of 
occurrences of the 1:1, 3:2, 2:1 resonances as the eccentricity varies in the interval $[0,0.4]$, while the dissipative parameter takes the values $K=0,10^{-4}, 10^{-3}, 10^{-2}$. We considered the orbits with initial conditions $x=0$ and $y$ varying over a sample of 1000 equally spaced points in the interval $[0.5,2.5]$. We interpret such results as follows. In general we obtain that the number of occurrences of each resonance increases as the dissipation gets stronger. Moreover, the resonances behave differently as the eccentricity is varied: the 1:1 resonance occurs less frequently as the eccentricity gets larger; an optimal value of the eccentricity is observed in the remaining cases: for example, concerning the 3:2 resonance, the number of occurrences is bigger at $e=0.05$ for $K=0$, while for $K=10^{-2}$ it is larger at $e=0.2$, thus showing a strong dependence of the non-synchronous resonances upon the orbital eccentricity.

\section{Conclusions}

A major question in the context of the spin-orbit interaction concerns the different behaviors of the Moon and of Mercury, and in particular the investigation of the probability of capture in the 1:1 or 3:2 resonances. We introduced a conservative model under quite general assumptions, and then we discussed the contribution of dissipative torques as provided by the standard MacDonald's and Darwin's formulations. The investigation of the dynamics has been performed through different techniques, like the frequency analysis and the computation of the maximum Lyapunov indicator. The results show that the occurrence of a specific resonance depends on several factors. First, the value of the orbital eccentricity: for small eccentricities the synchronous resonance prevails, while the 3:2 resonance is most likely to occur for higher eccentricities. Secondly, a dominant role is played by the dissipation factor, since the occurrence of resonances highly increases 
as the dissipation gets stronger. A comparative analysis of the different techniques (i.e., frequency analysis and Lyapunov indicators) clearly indicates that the relatively high eccentricity of Mercury might be responsible for its capture into a 3:2 resonance; on the other hand the lower eccentricity of the Moon might have leaded our satellite to select the synchronous resonance.

\section{A Integration algorithms}

The integration of the conservative problem can be naturally performed through some symplectic algorithms, like the modified Euler's method adopted throughout the text or a more accurate technique, like the fourth order symplectic integration developed in [19], [20] to which we refer as Yoshida's method. Nevertheless the choice of the integration algorithm for dissipative systems is not equally obvious. To this end we compare three different techniques: beside the Euler and Yoshida symplectic methods we consider also a 4th-order Runge-Kutta algorithm. In order to compare these methods we perform some numerical integrations by varying the parameters and the initial conditions, while the step-size is kept fixed for all methods (actually equal to 0.01). In all cases we considered we always found a good agreement among the three methods; this result led us to perform our integrations through the easier and faster technique, namely the modified Euler's method.

To give concrete examples we propose two sets of parameters and data, the first one leading to capture into the synchronous resonance (for $\varepsilon=10^{-2}, K=10^{-4}, x_{0}=0$, $\left.y_{0}=0.8\right)$ and the second one not yet trapped by a resonance $\left(\varepsilon=10^{-2}, K=10^{-6}\right.$, $\left.x_{0}=0, y_{0}=0.5\right)$. In both cases the comparison is provided by Figure $A .1$ which shows a very good agreement among the three algorithms, thus encouraging us to use the 
modified Euler's method (eventually adopting an adequate time-step), since it provides a fast and sufficiently accurate integration of the equations of motion.

\section{References}

[1] A. Celletti, Analysis of resonances in the spin-orbit problem in Celestial Mechanics: The synchronous resonance (Part I), Journal of Applied Mathematics and Physics (ZAMP) $4 \mathbf{1}$ (1990), 174-204.

[2] A. Celletti, and L. Chierchia, Hamiltonian stability of spin-orbit resonances in Celestial Mechanics, Cel. Mech. and Dyn. Astr. 76 (2000), 229-240.

[3] A. Celletti, C. Froeschlé and E. Lega, Dissipative and weakly-dissipative regimes in nearlyintegrable mappings, Discrete and Continuous Dynamical Systems - Series A 16, n. 4 (2006), $757-781$.

[4] A.C.M. Correia, and J. Laskar, Mercury's capture into the 3/2 spin-orbit resonance as a result of its chaotic dynamics, Nature 429 (2004), 848-850.

[5] G. Darwin, Tidal friction and cosmogony, Scientific papers, Cambridge University Press 2 (1908).

[6] S. D'Hoedt, and A. Lemaitre, The Spin-Orbit Resonant Rotation of Mercury: A Two Degree of Freedom Hamiltonian Model, Cel. Mech. Dyn. Astr. 89, n. 3 (2004), 267-283.

[7] P. Goldreich, Final spin states of planets and satellites, Astronom. J. 71, n. 1 (1966), 1-7.

[8] P. Goldreich, and S. Peale, Spin-orbit coupling in the solar system, Astronom. J. 71, n. 6 (1966), 425-438.

[9] M. Guzzo, E. Lega, C. Froeschlé, On the numerical detection of the effective stability of chaotic motions in quasi-integrable systems, Physica D 163 (2002) 1-25. 
[10] A.P. Itin, A.I. Neishtadt, A.A. Vasiliev, Captures into resonance and scattering on resonance in dynamics of a charged relativistic particle in magnetic field and electrostatic wave, Physica D 141, no. 3-4 (2000), 281-296.

[11] J. Laskar, Frequency analysis for multi-dimensional systems. Global dynamics and diffusion, Physica D 67 (1993), 257-281.

[12] J. Laskar, C. Froeschlé and A. Celletti, The measure of chaos by the numerical analysis of the fundamental frequencies. Application to the standard mapping, Physica D 56 (1992), $253-269$.

[13] G.J.F. MacDonald, Tidal friction, Rev. Geophys. 2 (1964), 467-541.

[14] C.D. Murray, and S.F. Dermott, Solar system dynamics, Cambridge University Press (1999).

[15] A.I. Neishtadt, Capture into resonance and scattering by resonances in two-frequency systems, (Russian) Tr. Mat. Inst. Steklova 250 , Differ. Uravn. i Din. Sist. (2005) 198-218; translation in Proc. Steklov Inst. Math., no. 3 (250) (2005) 183-203.

[16] A.I. Neishtadt, Averaging, capture into resonances and chaos in nolinear systems, Chaos (Woods Hole, MA, 1989), 261-273, Amer. Inst. Phys., New York (1990).

[17] S.J. Peale, The free precession and libration of Mercury, Icarus 178 (2005), 4-18.

[18] A. Wolf, J.B. Swift, H.L. Swinney and J.A. Vastano, Determining Lyapunov exponents froma time series, Physica 16D (1985), 285-317.

[19] H. Yoshida, Construction of higher order symplectic integrators, Physics Letters A 150, n. $5,6,7$ (1990), 262-268.

[20] H. Yoshida, Recent progress in the theory and application of symplectic integrators, Cel. Mech. Dyn. Astr. 56 (1993), 27-43. 

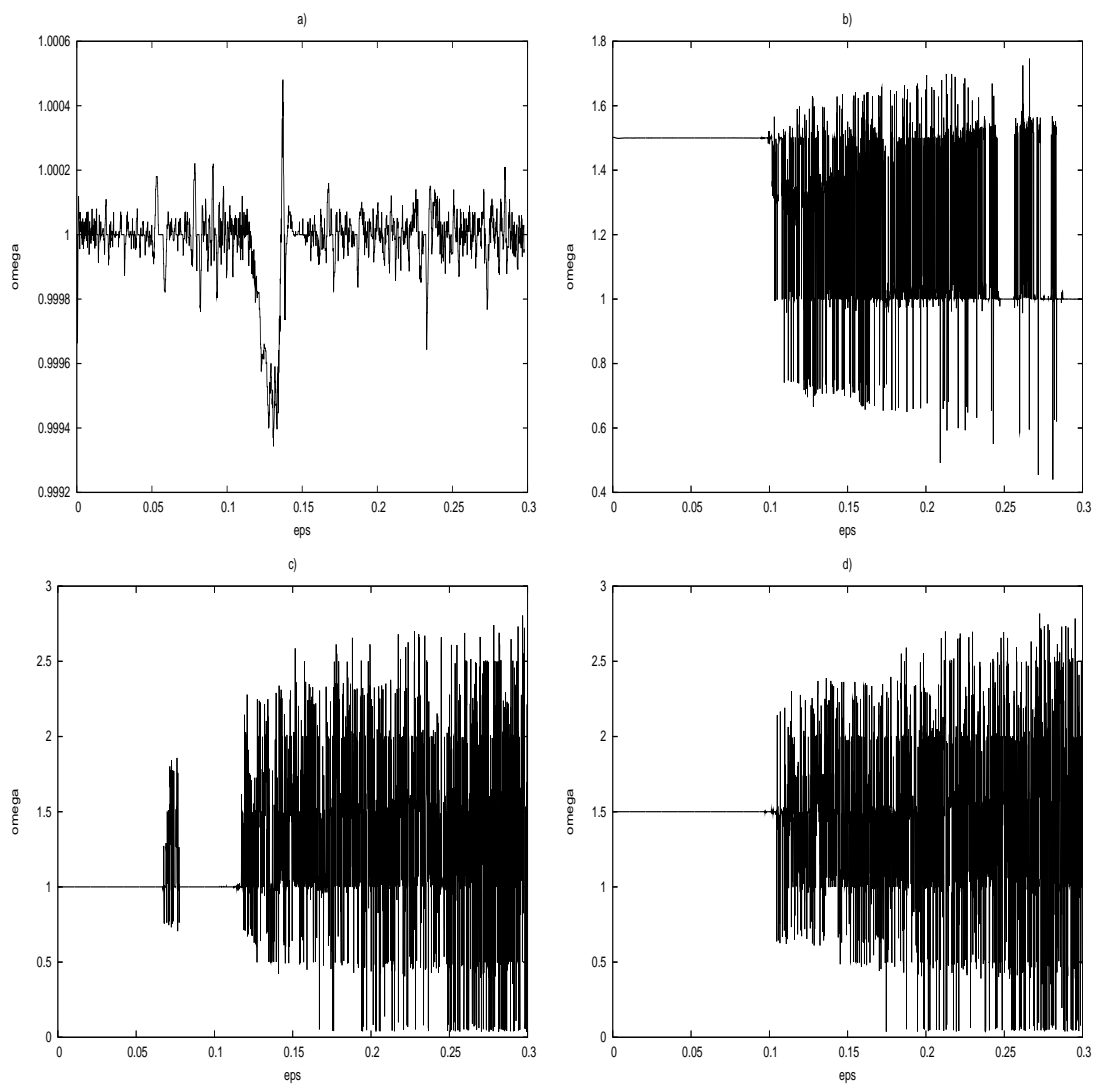

Fig. 1. Frequency analysis showing $\omega$ versus $\varepsilon$ in the conservative case. $a)$ Moon $(e=0.0549)$ with initial datum $y=1, x=0 . b)$ Moon $(e=0.0549)$ with initial datum $y=1.5, x=0 . c)$ Mercury $(e=0.2056)$ with initial datum $y=1, x=0 . d)$ Mercury $(e=0.2056)$ with initial datum $y=1.5, x=0$. 

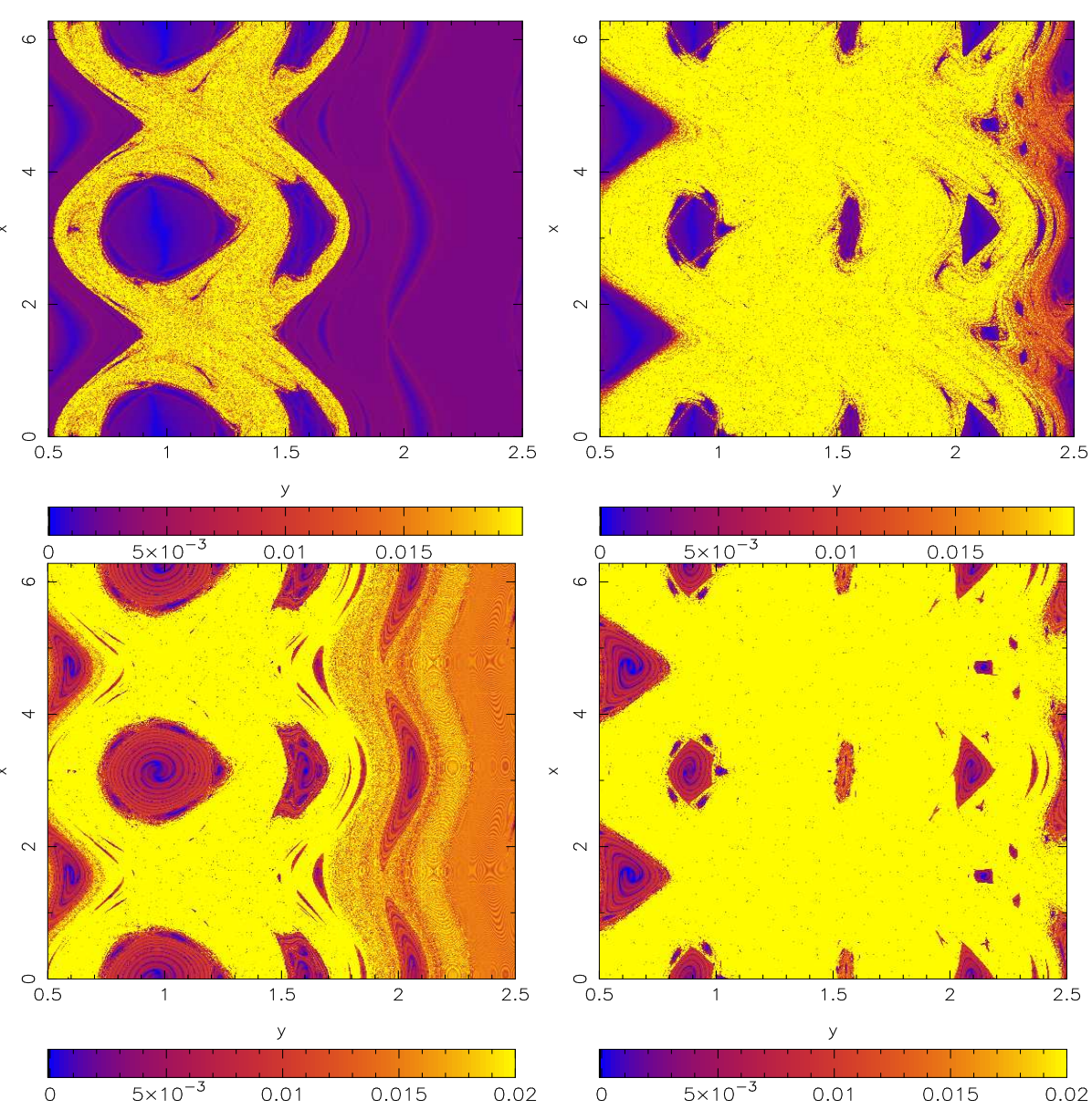

Fig. 2. Charts showing the maximum Lyapunov indicator in the $y$ - $x$ plane for $\varepsilon=0.1$. Top left: Moon, $K=0$; top right: Mercury, $K=0$; bottom left: Moon, $K=10^{-4}$; bottom right: Mercury, $K=10^{-4}$. 

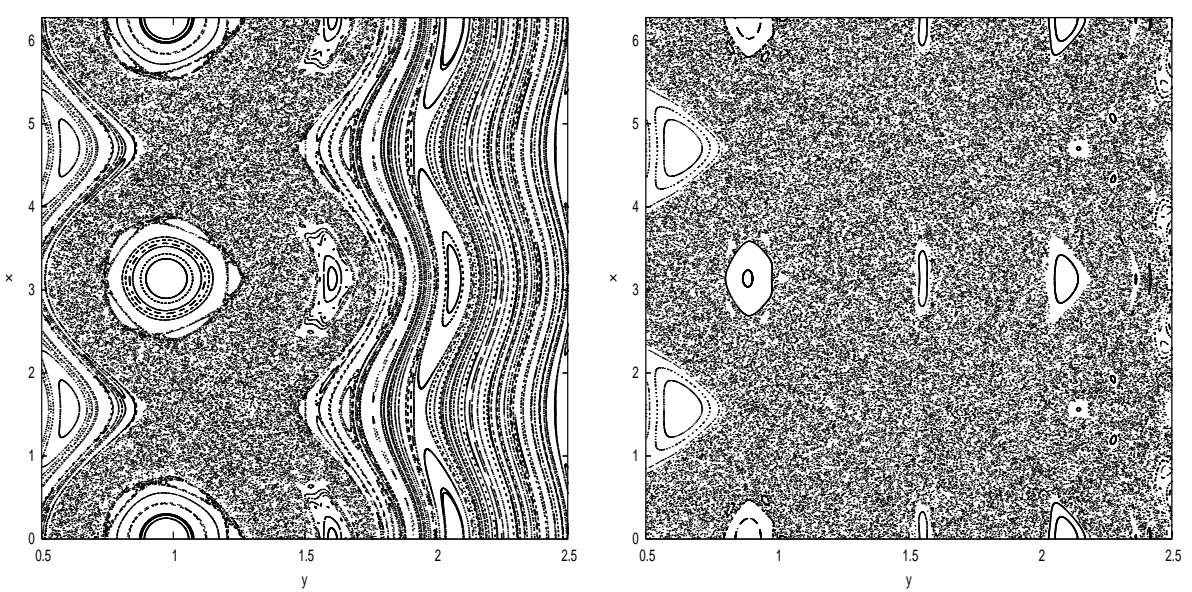

Fig. 3. Poincaré maps at times multiples of $2 \pi$ in the $y-x$ plane for $\varepsilon=0.1$. Top left: Moon, $K=0$; top right: Mercury, $K=0$.
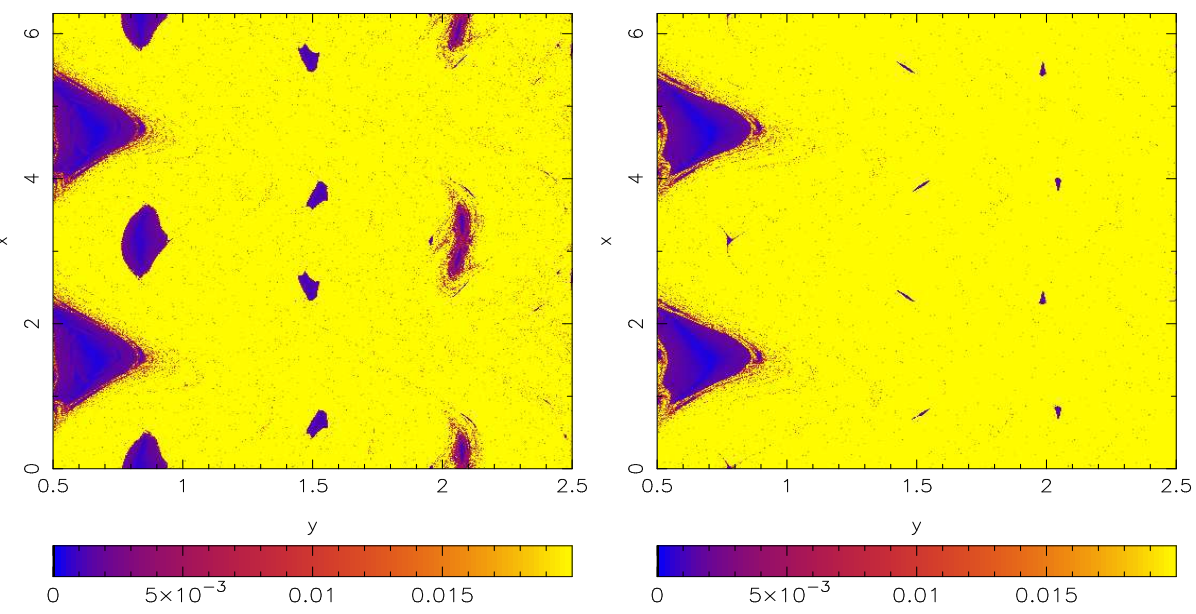

Fig. 4. Charts of the maximum Lyapunov indicator in the $y$ - $x$ plane for $\varepsilon=0.1, K=0$. Left: $e=0.3$; right: $e=0.4$. 

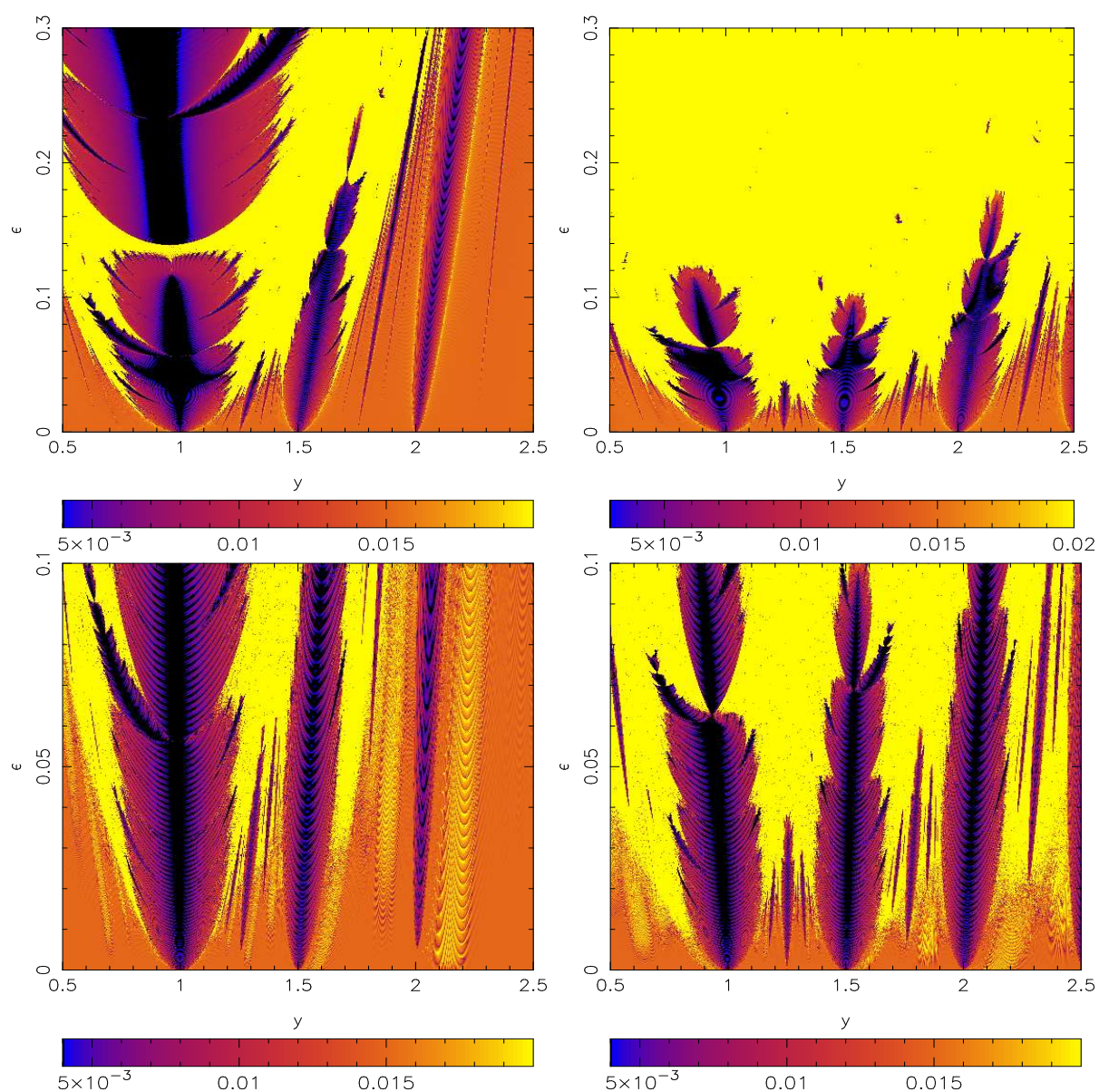

Fig. 5. Charts of the maximum Lyapunov indicator in the $y-\varepsilon$ plane for $x=0$. Top left: Moon for $K=0$; top right: Mercury for $K=0$; bottom left: Moon for $K=10^{-4}$; bottom right: Mercury for $K=10^{-4}$. 

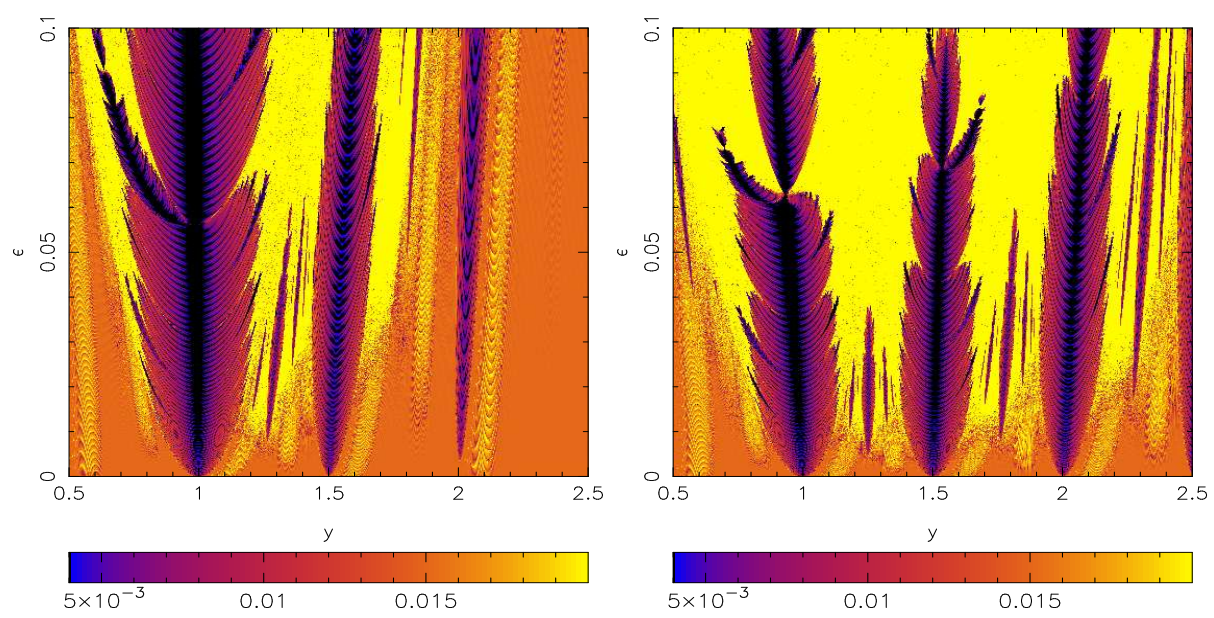

Fig. 6. Charts of the maximum Lyapunov indicator in the $y-\varepsilon$ plane for $x=0, K=10^{-4}$; Darwin's form of the dissipation has been considered. Left: Moon; right: Mercury. 

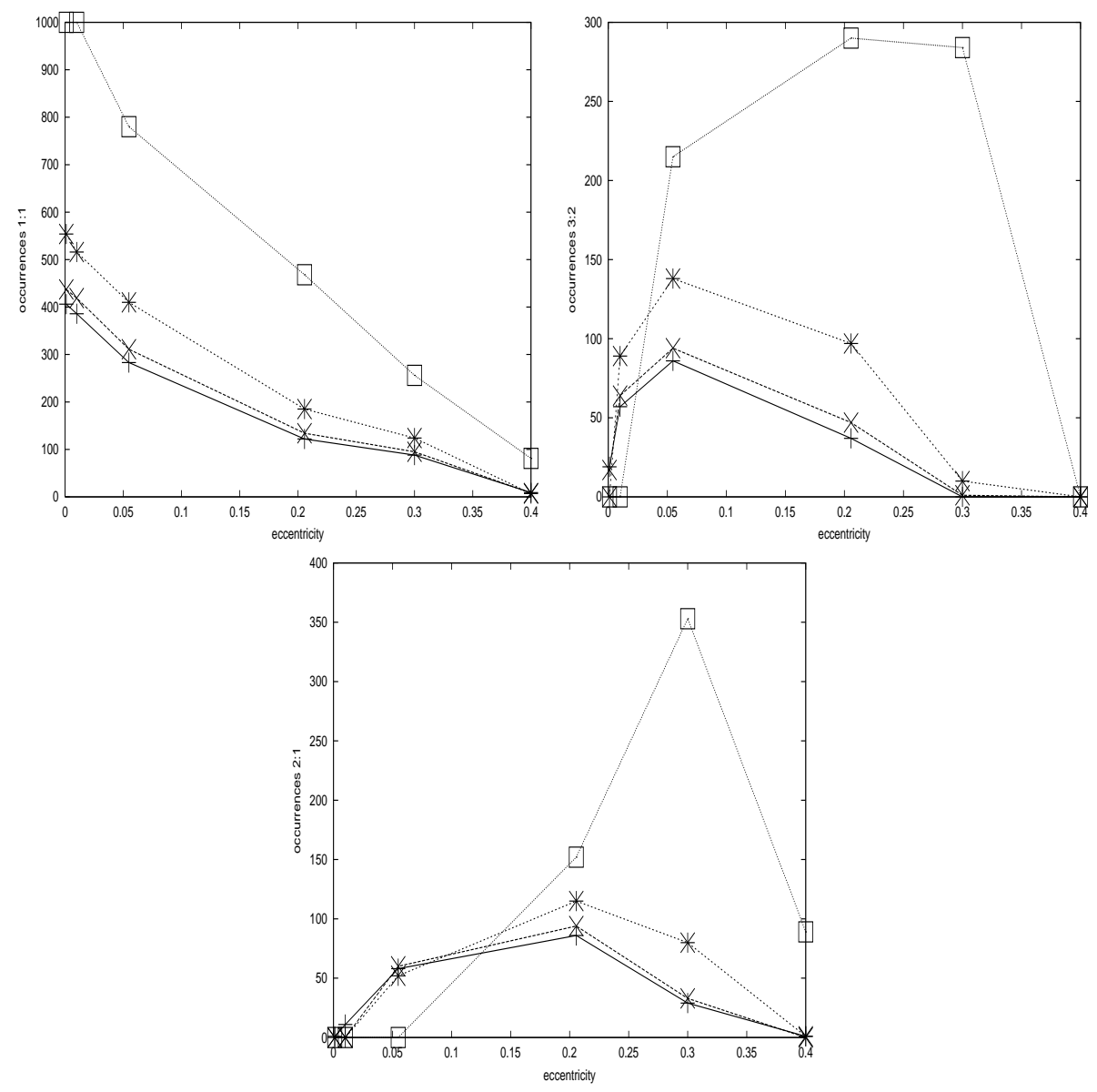

Fig. 7. Number of occurrences in a $p: q$ resonance versus the eccentricity, for different values of the dissipative parameter: + stands for $K=0, \times$ stands for $K=10^{-4}, *$ for $K=10^{-3}$, while the square stands for $K=10^{-2}$. Top left: 1:1 resonance; top right: 3:2 resonance; bottom: 2:1 resonance. 

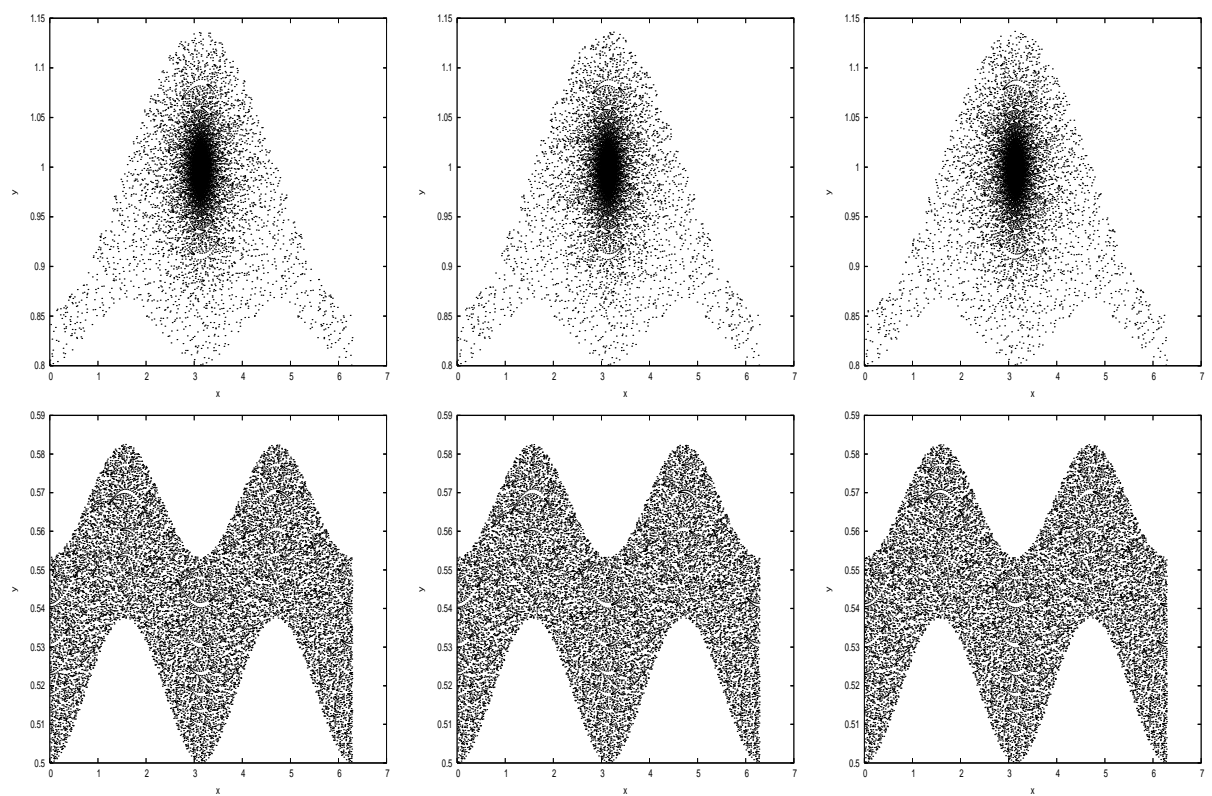

Fig. A.1. A comparison among a symplectic Euler's method (first column), Yoshida's fourth-order algorithm (second column) and a fourth-order Runge-Kutta integration (third column). In the first row the data are $\varepsilon=10^{-2}, K=10^{-4}, x_{0}=0, y_{0}=0.8$; in the second row the data are $\varepsilon=10^{-2}, K=10^{-6}, x_{0}=0, y_{0}=0.5$. 